\title{
Electron Transport and the Critical Temperature Gradient
}

\author{
W. Horton, G.T. Hoang, ${ }^{*}$ C. Bourdelle, ${ }^{*}$ X. Garbet,* M. Ottaviani, ${ }^{*}$ L. Colas* \\ Institute for Fusion Studies, The University of Texas at Austin, Austin, Texas 78712, USA \\ *Association Euratom-CEA, CEA/DSM/DRFC, CEA Cadarache, \\ 13108 Saint-Paul-Lez-Durance, France
}

\begin{abstract}
Tore Supra electron thermal fluxes, analyzed over a range of heating powers and plasma densities, are shown to vary parametrically according to the small-scale electron temperature gradient (ETG) model, rather than the ion inertial scale electrostatic gyro-Bohm model. Steady-state power balance analysis and time-varying interpretative transport simulations, performed on the Tore Supra Fast Wave Electron Heating (FWEH) database, validate the ETG thermal flux-versus-gradient relation and the existence of a critical electron temperature gradient. The critical gradient length $\mathrm{R} / \mathrm{L}_{\mathrm{c}}$ and the parametric dependence of the thermal flux, $\mathrm{q}_{\mathrm{e}}\left(\mathrm{n}_{\mathrm{e}}, \mathrm{T}_{\mathrm{e}}, \nabla \mathrm{T}_{\mathrm{e}}, \mathrm{q}, \mathrm{s}\right)$, agree well with the ETG model.
\end{abstract}




\section{Introduction}

Linear kinetic stability theory for electron and ion temperature gradient (ETG and ITG) instabilities yields a complicated set of growth-rate formulas, depending on the dominant plasma regime, with respect to the trapped particle collisionality, and the magnetic shear profile for tokamaks [1-6]. In addition, fluid simulations suggest that self-generated sheared flows may shift the effective critical gradient from the linear value [7,8]. Kinetic simulations provide a wealth of information on the linear and nonlinear behavior of both forms of turbulence [9-11]. Here, we examine the experimental evidence for the value and scaling of the critical temperature gradient, $\left(\nabla \mathrm{T}_{\mathrm{e}} / \mathrm{T}_{\mathrm{e}}\right)_{\mathrm{c}}$, in a well-documented multi-megawatt electron power flux qe database from Tore Supra [13-17]. The experimental scaling derived from unconstrained empirical power law formulas are compared with theoretically-derived values of the critical gradient length $R / L_{c}=\left(R \nabla T_{e} / T_{e}\right)_{c}$. The profiles are always above the critical gradient with the excess over critical increasing toward the plasma edge. The power balance scaling analysis of the database is found to be consistent with the high-plasma $\mathrm{m}_{\mathrm{i}} \beta_{\mathrm{e}} / \mathrm{m}_{\mathrm{e}}$ electron temperature-gradient-driven turbulence scaling of the electron thermal diffusivity [16-17]. In detail, the Fast Wave Heating $T_{e}>T_{i}$ discharges in Tore Supra show a local scaling of the turbulent diffusivity, $\chi_{e}$, with electron temperature that is shown to be consistent with $T_{e}^{1 / 2} / n_{e}$. The gyroBohm $\chi_{\mathrm{e}}$ scaling, $\mathrm{T}_{\mathrm{e}}^{3 / 2} / \mathrm{B}^{2} L_{T_{e}}$, gives too rapid an increase of the heat flux $\mathrm{q}_{\mathrm{e}}$, with $\mathrm{T}_{\mathrm{e}}$ and density $\mathrm{n}_{\mathrm{e}}$ to be consistent with the database. This finding is consistent with the large values of $m_{i} \beta_{e} / m_{e}=40$ in these low-field, hot helium plasmas.

Turbulent thermal transport is a universal mechanism leveling thermal gradients in neutral fluids, ionospheres, and hot magnetized plasmas. Near criticality, a laminar convection begins the transport process. As the temperature gradient increases, the fluid becomes turbulent, with large turbulent thermal fluxes carrying the imposed heat flux. How far past the critical gradient the system settles depends on many factors, including for example, the type of zonal flows and magnetic shear in the system. In nearly collisionless plasmas created through high-power auxiliary heating sources, it is natural to expect temperature-gradient-driven turbulent convection. Artsimovitch reported an anomalous electron thermal diffusivity at the time of the breakthrough with his first tokamak experiment [18]. Contemporary evidence for turbulent electron convection in high-power electron heating is ubiquitous, and seriously impedes the pathway to fusion power. Tore Supra, FTU [19] and ASDEX-U [20] provide well-documented examples of this anomalous electron transport. In Tore 
Supra (hereafter TS), up to 7MW of Fast Wave ion cyclotron radio frequency heating drives up a steep electron temperature gradient with $R \mid \nabla \mathrm{T}_{\mathrm{e}} \mathrm{l} \gg \mathrm{T}_{\mathrm{e}}$, where $\mathrm{R}$ is the major radius of the torus [1517]. The electron temperature gradient provides a reservoir of thermal energy to drive turbulent convection. The turbulent energy density, $\mathrm{W}$, is bounded above by the work $\mathrm{W}_{\mathrm{C}}$ done by a Carnot cycle operating between the temperature difference over the radial correlation scale length $\ell_{\mathrm{c}}$. Using the entropy formula for the electron gas, this upper bound gives $\mathrm{W}<\mathrm{W}_{\mathrm{C}}$, where $\mathrm{W}_{\mathrm{C}}=\mathrm{n}_{\mathrm{e}}$ $\ell_{c}^{2}\left(\mathrm{dT}_{\mathrm{e}} / \mathrm{dr}\right)\left[\mathrm{dT}_{\mathrm{e}} / \mathrm{T}_{\mathrm{e}} \mathrm{dr}-2 / 3 \mathrm{n}_{\mathrm{e}} / \mathrm{dn}_{\mathrm{e}} / \mathrm{dr}\right]$. Changing to the ion scale, the same formula applies with interchange of ions and electrons. Equating the released gradient energy with wave kinetic energy leads to the two growth rates and $\gamma_{\mathrm{e}}=\mathrm{v}_{\mathrm{e}} / L_{T_{e}}$ and $\gamma_{\mathrm{i}}=\mathrm{v}_{\mathrm{i}} / L_{T_{i}}$, well past the critical gradients [6]. Thus, we observe the sub-microsecond time scale for the small-scale turbulence compared with tens of microseconds time scale for the TEM-ITG turbulence. Nonlinear simulations [8-11] show that the space scale of the ETG turbulence gives a correlation length, such that the electron transport is comparable to the ion transport. When $\mathbf{E} \times \mathbf{B}$ sheared flows are present, the large-scale turbulence is more strongly reduced than the weakly-effected ETG turbulent transport [8-11]. The reversed shear transport barrier regime in JET produced in LHCD experiments has been found to be in such a regime [6].

Thus, we know that for electron transport there are two interacting pathways for turbulent electron thermal transport. The details of the $\mathbf{E} \times \mathbf{B}$ and magnetic shear determine which pathway dominates. The density and temperature scalings of the regimes differ. Only the trapped electrons participate in the low-frequency component of the turbulent thermal fluxes from the ITG-TEM fluctuations. The ETG correlation length is much larger than the electron gyroradius, and can be comparable to the electron collisionless skin depth, $\delta_{\mathrm{s}}=\mathrm{c} / \omega_{\mathrm{pe}}$, for higher electron $\beta_{\mathrm{e}}=2 \mu_{0} \mathrm{p}_{\mathrm{e}} / \mathrm{B}^{2}$ values.

In Table 1 we give formulas for the critical gradient for driving the turbulence. From the ideal Carnot cycle, the critical temperature gradient is derived from the entropy gradient of the electron gas, which is traditionally expressed in terms of the critical $\eta_{\mathrm{e}}=\nabla \ell n \mathrm{~T}_{\mathrm{e}} / \nabla \ell n \mathrm{n}_{\mathrm{e}}>2 / 3$ (and $\eta_{i}>2 / 3$ for ITG in the ion gas). These formulas are shown in the first row in Table I. The presence of magnetic shear, $\mathrm{s}=\mathrm{rq} / \mathrm{q}$, forces the drift waves into regions of electron [ion] Landau damping, which increases the threshold gradient with a linear dependence on Is/ql $\left(1+Z_{\text {eff }} T_{e} / T_{i}\right)\left[\right.$ and $\left(1+T_{i} / T_{e}\right)$ for ITG], shown in the second row of Table I. When the density profile is flat, and the toroidal gradient- 
curvature drift resonance dominates electron-wave interactions, the linear threshold changes again, to be given by the third row of Table I. The fourth row of Table I gives composite formulas found through gyrokinetic simulations [11]. While for $\mathrm{T}_{\mathrm{e}}=\mathrm{T}_{\mathrm{i}}$ the critical gradient for ITG equals that of the ETG, there, $T_{e} / T_{i}$ dependence is strong. For ITG, the threshold is $R / L_{T i}=1.88 \mathrm{Is} / \mathrm{ql}\left(1+\mathrm{T}_{\mathrm{i}} / \mathrm{T}_{\mathrm{e}}\right)$ [4]. The physics behind this change is that it is the response of the adiabatic species that sets the threshold for the critical gradient, as most easily seen from the Nyquist diagram analysis of the dispersion relation [1], and the adiabatic species changes from ions to electrons in going from the ETG to the ITG instability. Jenko et al. [11] give a complex formula given in Table 1 for the switching from density gradient to shear control of the critical gradient. Dong et al. [3] give a complex set of functions describing the details of the shear and q dependence of the critical gradient and the growth rates derived from the integral eigenvalue problem for ETG drift waves and discuss the relation to the Jenko et al. formula. Experimental findings on electron transport for eight tokamaks (ASDEX-U, COMPASS-D, RTPm TCV, FT-U, AUG, JET and Tore Supra) are discussed in the review article of Ryter et al. [20].

Here we show direct experimental evidence in Tore-Supra, for the presence of the magnetic shear control of the critical-electron-temperature gradient (shown in row 2 of Table I). There is an offset term of $2-3 \mathrm{keV} / \mathrm{m}$ arising in the data derived critical electron temperature consistent with the ETG formulas.

In previous electron heating experiments, the existence of such a threshold is suggested by the electron transport analysis [13-17] and by the measurements of both density and magnetic fluctuations [21-24]. Examples of rising levels of magnetic fluctuations [22], with increasing midradius electron temperature gradients, are shown in Fig. 1. The scattering wavenumber is fixed at $\mathrm{k}_{\mathrm{r}}=12 \mathrm{~cm}^{-1}$ by the geometry and frequency. For a local electron density of $3 \times 10^{19} \mathrm{~m}^{-3}$, the collisionless skin depth $\delta_{\mathrm{s}}=\mathrm{c} / \omega_{\mathrm{pe}}=1 \mathrm{~mm}$ and $\mathrm{k}_{\mathrm{r}} \mathrm{c} / \omega_{\mathrm{pe}}=1.2$. The scattering data is not available for the particular FWEH discharges used in the power balance studies, therefore, a quantitative comparison is not possible. Similar discharges with scattering data are available [21-24]. Extrapolation of the density fluctuation level, versus temperature gradient to the intercept of zero fluctuation amplitude, also yields a finite-threshold gradient of the order of a few $\mathrm{keV} / \mathrm{m}$. The internal magnetic fluctuation levels determined by the cross-polarization scattering (CPS) experiments are known with less confidence. Extrapolation of the magnetic fluctuation to zero may be consistent with the ETG threshold in $\nabla \mathrm{T}_{\mathrm{e}}$. Magnetic fluctuations, however, are driven by gradients in the current density profile, as well as the electron temperature profile. Wong et al. [25] has reported that for short 
wavelength, $\mathrm{k}_{\perp}=9 \mathrm{~cm}^{-1}\left(\mathrm{k}_{\perp} \rho_{\mathrm{i}} \sim 5\right.$ and $\left.\mathrm{k}_{\perp} \mathrm{c} / \omega_{\mathrm{pe}} \sim 0.8\right)$, density fluctuations in the Tokamak Fusion Test Reactor (TFTR) [26] are correlated with large $\chi_{\mathrm{e}}$ in the ERS discharges. Small skin depth scale magnetic fluctuations called microtearing modes, may also be present and nonlinearly coupled to the ETG turbulence.

In Sec. II the theoretical formulas used in the analysis are given, including the threshold gradient formulas, and the turbulent correlation lengths. Section III is dedicated to the analysis of the Fast Wave Electron Heating (FWEH) database. In particular, the dependence of the radial electron thermal flux $\mathrm{q}_{\mathrm{e}}$ on the temperature gradients, is studied in detail in Sec. III.B, while in Sec. III.C the parametric dependence of the observed critical gradient length is given, and compared with the theoretical formulas. Conclusions are presented in Sec. IV.

\section{Analysis Method for the Critical Gradient}

The critical gradient $\left(\nabla \mathrm{T}_{\mathrm{e}}\right)_{\mathrm{c}}$ is defined in terms of the dependence of the local electron thermal flux $\mathrm{q}_{\mathrm{e}}$ and the local temperature gradient through the theoretical formulation

$$
\mathrm{q}_{\mathrm{e}}=-\mathrm{n}_{\mathrm{e}} \chi_{\mathrm{e}}\left[\left(\nabla \mathrm{T}_{\mathrm{e}}\right)-\left(\nabla \mathrm{T}_{\mathrm{e}}\right)_{\mathrm{c}}\right] \text {. }
$$

The formulas for the thermal diffusivity in Eq. (1) follow from turbulence theory, in which the turbulent diffusivity, $\chi_{\mathrm{e}}(\mathrm{W})$, is proportional to the turbulent energy density, $\mathrm{W}(\mathrm{r}, \mathrm{t})$. Depending on the speed of the radial propagation, the turbulence level, W(r), is small to vanishing in regions where the gradient $\left(\nabla \mathrm{T}_{\mathrm{e}}\right)$ is below the linear theory critical gradient $\left(\nabla \mathrm{T}_{\mathrm{e}}\right)_{\mathrm{c}}$ for electron temperature gradient (ETG) turbulence. Nonlinear simulations and test particle integrations support the turbulent

conductivity formula, $\chi_{\mathrm{e}}=\ell_{\mathrm{c}}^{2} \gamma^{\max }$, where $\ell_{\mathrm{c}}$ is the radial correlation length, and $\gamma^{\max }$ is the local maximum linear rate. From theory, there is a transition between two correlation length regimes, as the plasma electron beta increases. For high $\mathrm{m}_{\mathrm{i}} \beta_{\mathrm{e}} / \mathrm{m}_{\mathrm{e}}$, the turbulence is electromagnetic and the electrons are chaotic from both the $\mathbf{E} \times \mathbf{B}$ drifts and the magnetic flutter [27]. Here, simulations show that collisionless skin depth $\delta_{\mathrm{s}}=\mathrm{c} / \omega_{\mathrm{pe}}$ is the key scale length for the electron decorrelation in the electromagnetic fluctuations, in the limit of high $\mathrm{m}_{\mathrm{i}} \beta_{\mathrm{e}} / \mathrm{m}_{\mathrm{e}}$. For small $\mathrm{m}_{\mathrm{i}} \beta_{\mathrm{e}} / \mathrm{m}_{\mathrm{e}}$, the magnetic fluctuation is negligible, and the electrostatic ETG fluctuations develop an extended radial structure that leads to substantial $\mathbf{E} \times \mathbf{B}$ transport. The heat flux formulas are

$$
\mathrm{q}_{\mathrm{e}}=-\mathrm{n}_{\mathrm{e}} \mathrm{f}_{\text {tre }} \chi^{\mathrm{ITG}} \nabla \mathrm{T}_{\mathrm{i}}=\mathrm{C}_{\mathrm{e}}^{\mathrm{ITG}} \mathrm{n}_{\mathrm{e}} \mathrm{T}_{\mathrm{i}}(\mathrm{r} / \mathrm{R})^{1 / 2} \frac{c q^{2} \rho_{s}^{2}}{L_{T_{i}} R}\left(\frac{R}{L_{T_{i}}}-\frac{R}{L_{c}}\right)
$$




$$
\begin{aligned}
& \mathrm{q}_{\mathrm{e}}=-\mathrm{n}_{\mathrm{e}} \chi_{e}^{e m} \nabla T_{e}=C_{e}^{e m} n_{e} T_{e} q \frac{c^{2}}{\omega_{p e}^{2}} \frac{\mathrm{v}_{\mathrm{e}}}{\mathrm{R}^{2}}\left(\frac{R}{L_{T_{e}}}-\frac{R}{L_{c}}\right) \text { for } \beta_{\mathrm{e}}>\beta_{\mathrm{e}, \mathrm{cr}} \\
& \mathrm{q}_{\mathrm{e}}=-\mathrm{n}_{\mathrm{e}} \chi_{e}^{e s} \nabla T_{e}=C_{e}^{e s} n_{e} T_{e} q^{2}\left(\frac{\rho_{e}^{2} \mathrm{v}_{e}}{L_{T e}^{2}}\right)\left(\frac{R}{L_{T e}}-\frac{R}{L_{c}}\right) \quad \text { for } \quad \beta_{\mathrm{e}}<\beta_{\mathrm{e}, \mathrm{cr}}
\end{aligned}
$$

where $\mathrm{f}_{\mathrm{tr}}$ is the trapped electron fraction and $\beta_{\mathrm{e}, \mathrm{cr}}=\left(L_{T_{e}} / \mathrm{qR}\right)^{2}$ or $\alpha_{\mathrm{MHD}} \sim L_{T_{e}} / \mathrm{R}[1,28,29]$. At this critical value $\beta_{\mathrm{e}, \mathrm{cr}}$, or $\alpha_{\mathrm{MHD}}$, the fluxes (2b) and (2c) are equal. Typical values are $\beta_{\mathrm{e}} \cong 0.01\left(\mathrm{p}_{\mathrm{e}}=30 \mathrm{kPa}\right.$ and $\mathrm{B}^{2} / 2 \mu_{0}=1.9 \mathrm{MPa}$ at $2.2 \mathrm{~T}$ ) in helium working gas, so that $\mathrm{m}_{\mathrm{i}} \beta_{\mathrm{e}} / 2 \mathrm{~m}_{\mathrm{e}}=40$ and $\beta_{\mathrm{e}}$ is well above $\beta_{\mathrm{e}, \mathrm{cr}}$ $=\left(L_{T_{e}} / \mathrm{qR}\right)^{2}=(.10 \mathrm{~m} / 3 * 2.3 \mathrm{~m})^{2}=2 \times 10^{-4}$.

The turbulent thermal fluxes in Eq. (2) vanish at the critical gradient $\left(\nabla \mathrm{T}_{\mathrm{e}}\right)_{\mathrm{c}}$, as expressed in Table 1. In reality, the flux would drop to a low value determined by the still longer wavelength resistive MHD fluctuations, and the residual flux due to electron-ion collisions (neoclassical guiding center flux). A description of the role of magnetic shear on the chaotic guiding-center orbits that produce the turbulent fluxes is given in Kim et al. [27].

One contrary indication to Eq. (2b) that remains to be explained is given in Ref. [28] in which the $\beta_{\mathrm{e}}$ variation of the local diffusivity in a fixed thermal flux 3D fluid simulation is tested. They [28] find a very weak decrease of the turbulent diffusivity with beta increasing from $10^{-3}$ to $10^{-2}$, and then rather constant diffusivity for beta increasing to $\max \left(\beta_{\mathrm{e}}\right)=0.04$. There are large, titled radial convection cells with aspect ratios between 2 to 3 in the steady state that is itself close to marginal stability. The resolution of this contrary result may require much larger space scale simulations that couple the short wavelength and long wavelength fluctuations more effectively through a complex region in the transitional behavior of the ion and electron dynamics.

\section{Power Balance Analysis and Interpretative Transport Simulations}

Electron transport is particularly sensitive to the magnetic field configuration, i.e., to the safety factor $\mathrm{q}$ and to the magnetic shear $\mathrm{s}=\mathrm{rq} / \mathrm{q}$. In order to isolate this magnetic topology dependence, experiments were chosen where sawteeth were absent or weak, and the electron heat source was well localized to the core plasma of Tore Supra [12,31]. The electron transport analysis of these FWEH discharges allows an accurate experimental determination of the radial profile of the thermal flux $\mathrm{q}_{\mathrm{e}}$. The critical gradient is defined as the extrapolation following Eq. (1) to zero heat 
flux. This critical gradient is an inferred value, since the heat flux never vanishes in tokamak experiments.

\section{A. Description of FWEH database}

Figures 2, 3, and 4 are based on a series of 17 shots with increasing $T_{e}$ from $P_{F W}=0.75-7.4$ MW with the same density $n_{e}$ and q profiles in TS at plasma current $I_{p}=0.6$ MA and toroidal field $\mathrm{B}=2.2 \mathrm{~T}$. The range of the total plasma heating power is 1.5-7.5 MW, including the Ohmic power $\mathrm{P}_{\mathrm{OH}}=0.1-0.75 \mathrm{MW}$. From this data, 21 time slices in the stationary phases of the discharges were selected. Owing to the large variation of $\mathrm{P}_{\mathrm{FW}}$, the electron-temperature gradient increases from 1 $\mathrm{keV} / \mathrm{m}$ to $12 \mathrm{keV} / \mathrm{m}$ [Fig. 2(a)]. The power balance analysis is performed for normalized radii (r/a) between 0.2 and 0.75 . The q profiles are measured by the polarimetry (Faraday rotation), with an error in the range of $10 \%$ (at mid-radius) and $25 \%$ (at the center). The relative error of $\mathrm{T}_{\mathrm{e}}$ from 28 radial points, measured by Thomson scattering diagnostic (12 channels), and a superheterodyne radiometer (16 channels), is less than $20 \%$.

\section{B. Flux versus gradient analysis}

The power balance figures in Hoang et al. [17] show that the flux $\mathrm{q}_{\mathrm{e}}$ increases by a factor of about 10 from $\mathrm{q}_{\mathrm{e}}(\mathrm{r} / \mathrm{a}=0.2)=0.03 \mathrm{MW} / \mathrm{m}^{2}$ to $0.3 \mathrm{MW} / \mathrm{m}^{2}$ and $0.007 \mathrm{MW} / \mathrm{m}^{2}$ to $0.08 \mathrm{MW} / \mathrm{m}^{2}$ at $\mathrm{r} / \mathrm{a}=$ 0.75. The error bars of $\mathrm{q}_{\mathrm{e}}$ are principally due to the uncertainty of the electron-ion equipartition power transfer to the ions. Best fits for the extrapolation to zero of the flux versus the gradient, which are weakly dependent on $\mathrm{r}(0.50 \pm 0.15)$, give $\left(\nabla \mathrm{T}_{\mathrm{e}}\right)_{\mathrm{c}}$ between $2-3 \mathrm{keV} / \mathrm{m}$. The radial profile of $\left(\nabla \mathrm{T}_{\mathrm{e}}\right)_{\mathrm{c}}$ is rather flat. If the offset from the critical gradient is dropped, $\left(\nabla \mathrm{T}_{\mathrm{c}}=0\right)$, in Eq. (1), and $\mathrm{q}_{\mathrm{e}}$ is parameterized by a power law fit of the form $\mathrm{q}_{\mathrm{e}} \propto \mid \nabla \mathrm{T}_{\mathrm{e}} \mathrm{e}^{\gamma}$, one finds that the exponent $\gamma$ itself would have a strong radial variation increasing from $\gamma=1.5$ at $\rho=0.2$ to $\gamma=2.5$ at $\rho=0.8$. Thus, we must consider the presence of the critical gradient for parameterizing the flux.

Now we describe the dependence of $\mathrm{q}_{\mathrm{e}}$ on temperature and density that occurs for the parameterization, with the inferred critical gradient $(\nabla \mathrm{T})_{\mathrm{c}}$. We define the exponents $\alpha$ and $\beta$ through fitting the power balance $\mathrm{q}_{\mathrm{e}}$ data with the formula

$$
\mathrm{q}_{\mathrm{e}}=\text { const. } \mathrm{n}_{\mathrm{e}}^{\beta} \mathrm{T}_{\mathrm{e}}^{\alpha}\left(1 / \mathrm{L}_{\mathrm{Te}}-1 / \mathrm{L}_{\mathrm{c}}\right) \text {. }
$$

Figure 2(a) shows that there is a well-defined minimum deviation at all radii given by $\alpha=3 / 2$ and $\beta=$

0 . The relative deviation between the parameterized flux (4) and the power balance flux is about $15 \%$ 
and increases rapidly to $100 \%$ if the $\alpha$ is increased to the gyroBohm value of $5 / 2$. A fit with $\beta=0.5$ yields the unsatisfactory model that $\alpha$ must vary appreciable with radius to maintain a reasonable 15 $20 \%$ standard deviation.

At a given radius, $L_{T_{e}}$ has a weak variation (within the error bar), while $\mathrm{q}_{\mathrm{e}}$ increases by a factor of 10. Figure 2(b) shows the scatter of the normalized heat flux data from Eq. (4) for $\alpha=3 / 2$ and $\beta=0$. The variation of $1 / L_{T_{e}}$ is around $30 \%$ for $\mathrm{r} / \mathrm{a}<0.7$. The linear fit of the normalized heat flux, $y=q_{e}{ }^{*}=q_{e} / T_{e}{ }^{3 / 2}$ versus $x=R / L_{T^{-}} R / L_{c}$ in Fig. 2(b) gives a relative deviation of about $20 \%$ for all radii. Conversely, Fig. 2(c) shows that the relative deviations are much larger when the heat flux is proportional to the plasma density $(\beta=1)$. The deviations are between $1-3$, when $\mathrm{q}_{\mathrm{e}}$ is normalized to $\mathrm{n}_{\mathrm{e}} \mathrm{T}_{\mathrm{e}}^{5 / 2}$ (dashed line), according to electrostatic gyroBohm transport models: $\mathrm{q}_{\mathrm{e}}{ }^{\mathrm{gB}} \propto$ $\mathrm{n}_{\mathrm{e}} \mathrm{T}_{\mathrm{e}}^{5 / 2}\left(L_{T_{e}}^{-1}-L_{c}^{-1}\right)$ given in Eq. (2c), where $\alpha=5 / 2$ and $\beta=1$. For the hybrid formula where $\mathrm{q}_{\mathrm{e}}$ is normalized to $\mathrm{n}_{\mathrm{e}} \mathrm{T}_{\mathrm{e}}^{3 / 2}$ (dotted line), and the deviations increase to $40 \%$. The best parameterization of the data is clearly given by the exponents $\alpha=3 / 2$ and $\beta=0$ giving the solid line in Fig. 2(c). The results apply for the Greenwald fraction $\mathrm{n}_{\mathrm{e}} / \mathrm{n}_{\mathrm{GW}}=0.5$, and may not work well too close to the Greenwald density limit, or at a low-plasma beta.

Figure 3 shows an example of the variation of $1 / L_{T_{e}}$ during the application of $\mathrm{FW}$ power ramped up to $6 \mathrm{MW}$ in a discharge performed at $\mathrm{I}_{\mathrm{p}}=0.9 \mathrm{MA}$ and central line density around $7 \times$

$10^{19} \mathrm{~m}^{-2}$. The profiles of $T_{e}$ are measured by 14 channels of a superheterodyne radiometer giving accurate values of $\nabla \mathrm{T}_{\mathrm{e}}$ from the distance between two successive channels $(6 \mathrm{~cm})$. Relative increases of R/ $L_{T_{e}}$, when the total power increases from 0.7 MW (Ohmic phase) to 6.5 MW, confirm the results of the analysis of the database with Eq. (4). This trend is similar to the observation of the so-called 'stiff' $\mathrm{T}_{\mathrm{e}}$ profile in the ASDEX experiments [19,32], in which $L_{T_{e}}$ is almost constant when the heat flux varies by a factor of approximately 3 . The value of $\mathrm{C}^{\mathrm{em}} \simeq 0.1 \mathrm{in} \mathrm{Eq.} \mathrm{(2b)} \mathrm{is} \mathrm{found} \mathrm{to} \mathrm{describe} \mathrm{that}$ FWEH database. We discuss the accuracy of this conclusion now.

\section{Parametric dependence of $1 / \mathrm{L}_{c}$}

Next, the parametric dependence of the threshold length $\mathrm{L}_{c}$ has been studied. No significant dependences on $n_{e}, q$, and density gradient were observed. The offset value $R / L_{c}$ at a given radius, corresponding to two series of discharges characterized by completely different $\mathrm{n}_{\mathrm{e}}, \mathrm{L}_{\mathrm{n}}$ and $\mathrm{q}$ profiles, but the same ratio s/q, is practically unchanged from the data in Fig. 3. This suggests that the radial 
variation of $1 / \mathrm{L}_{\mathrm{c}}$, displayed in Fig. 3(a), is mainly related to the ratio s/q. The dimensionless parameter $\mathrm{R} / \mathrm{L}_{\mathrm{c}}$, deduced from both power balance and stability analyses with a linear electrostatic gyrokinetic code [5,6], is plotted versus s/q in Fig. 3(b) $(\mathrm{R}=2.28 \mathrm{~m}$, being the major radius), showing that $1 / \mathrm{L}_{\mathrm{c}}$ increases linearly with s/q. Critical values deduced from the calculation of the linear electrostatic growth rates are somewhat lower than the values obtained from the power balance analysis. This type of shift effect is found in ITG simulations, where it is sometimes called the Dimits shift [7]. In the case of ITG modes, the explanation of the shift is given by zonal flows that in the ETG case are a weaker effect. In conclusion, a best fit of the data of the power balance analysis gives an offset linear formula with

$$
\mathrm{R} / \mathrm{L}_{\mathrm{c}}=5( \pm 1)+10( \pm 2)|\mathrm{s}| / \mathrm{q} \text {. }
$$

This formula is consistent with improved confinement due to increasing magnetic shear Is| in the pressure gradient region, which is thought to partially explain the high-plasma inductance $\ell_{\mathrm{I}}$ improved confinement mode in tokamaks. A similar dependence on magnetic shear to the experimental law in Eq. (5) is predicted by the ETG models in Refs. [1, 3, 5, 6, 9]:

$$
\mathrm{R} / \mathrm{L}_{\mathrm{c}} \cong 1.9(\mathrm{|s} \mid / \mathrm{q})\left(1+\mathrm{Z}_{\mathrm{eff}} \mathrm{T}_{\mathrm{e}} / \mathrm{T}_{\mathrm{i}}\right)
$$

Formula (6) tracks the radial profile of the experimental threshold well [Fig. 3(a)]. The results of nonlinear gyrokinetic simulations of toroidal turbulence, driven by the ETG, give the same shearcontrolled dependence, as shown in Table 1. Formula [11] in the last row of the Table works well and contains the density gradient scale length, the ellipticity $\kappa$, and the inverse aspect ratio $\varepsilon=r / R$, in addition to the usual factors in Eq. (6).

\section{Integrated predictive simulations with ETG Transport}

Predictive simulations of FWEH discharges on Tore Supra, with an upgraded version of the CRONOS code [33], strongly favor the electromagnetic turbulent transport model given in Eq. (2b) confirming the earlier indications [14-17] of a good correlation of the ETG model with the electron power balance data. The CRONOS code solves, in a self-consistent manner, the coupled system of transport equations together with the plasma equilibrium, current diffusion and sources (i.e. heat, particle and non-inductive current) for multiple species plasmas. The integrated system code is 
required, due to the feedback and feed-forward couplings between transports, ray tracing, and RF power absorption of the profiles and loop voltages.

Figure 4 shows, in the top left panel, the programmed FW power pulse for shot TS 18369 , followed below by the evolution of the electron temperature at four radial positions (solid line) compared with the theoretical model (broken line). The comparison for the total stored energy is given (bottom left panel) and is found in excellent agreement, due to the reasonably good agreement of the radial profiles. In the top right panel of Fig. 4, the comparison of the predicted and measured loop voltage also shows good agreement. The polarimetry diagnostic measurement is given in the lower right-hand panels of Fig. 4. The coupled integrated simulation codes predict the current profile penetration from which the poloidal magnetic field and total field angle are compared with those

measured by the Faraday rotation diagnostic. Again the agreement is good. Thus, we may have confidence that the predictions of this integrated package of simulation codes are reliable for forecasting the results of new higher power experiments.

\section{Conclusions}

In summary, a critical electron temperature gradient is clearly shown to exist in Tore Supra plasmas, with a weak radial dependence, such that $\left(\nabla \mathrm{T}_{\mathrm{e}}\right)_{\mathrm{c}}=2-3 \mathrm{keVm}^{-1}$. The experimental result supports the critical gradient length thermal flux model given in Eq. (2b). No significant dependence of the heat flux, $\mathrm{q}_{\mathrm{e}}$, on the density is found, consistent with the electromagnetic ETG turbulence model, and opposed to the electrostatic gyro-Bohm formulas. The inverse density dependence agrees with the Kadomtsev $\chi_{e}^{e m}$ formula [18], but other dependences are different. The inverse dependence of $\chi_{\mathrm{e}}$ on density is expected to apply only to a limited range of $\mathrm{n}_{\mathrm{e}} / \mathrm{n}_{\mathrm{GW}}$ and $\beta_{\mathrm{e}}$. Low aspect ratio devices that have a large fraction of trapped particles may show additional transport from the trapped electron mode that can mask this base level ETG transport.

Other turbulence models do not give a comparable degree of agreement as shown by the parametric scans in Fig. 2. For example, the collisionless heat flux for the ITG-TEM branch is proportional to the electron density and has a stronger increase with electron temperature than found in the power balance $\mathrm{q}_{\mathrm{e}}$. The dissipative version of TEMs would have the observed density dependence but has too strong a temperature dependence. More importantly, the FWEH discharges are clearly out of the collisional regime and into the collisionless regime with $\nu_{*} \ll 1$ and $T_{e}>T_{i}$. Other tokamak discharges with neutral beam heating, for example, show electron transport closer to the electrostatic gyro-Bohm value. We thus frame the hypothesis that FWEH keeps the $\chi_{\mathrm{e}}$ value near 
to a theoretical base level of gyro-Bohm divided by $\beta_{\mathrm{e}}$ as proposed in several theories. For example, the current diffusion turbulence model advocated by Itoh et al. $[30,34]$ has the same density and temperature dependence as the electromagnetic ETG. Mathematically, these two instabilities (ETG and the current diffusion) are closely related, containing a nonlinear Ohm's law, in addition to the standard vorticity and thermal balance equations. ETG models [1] are intrinsically two-component kinetic plasma models, as required for collisionless plasmas.

Above the critical threshold, the electron heat transport strongly increases: the variation of critical gradient length $\mathrm{R} / \mathrm{L}_{\mathrm{c}}$ is weak $(\approx 30 \%)$, while the heat flux increases by a factor of 10 . This rapid increase of $\mathrm{q}_{\mathrm{e}}$ with $\nabla \mathrm{T}_{\mathrm{e}}$ explains why many strong power dependence models $\left(\nabla \mathrm{T}_{\mathrm{e}}\right)^{\gamma}$; without the offset term in Eq. (2), can simulate electron transport with acceptable discrepancies when the temperature gradient exceeds the critical value. (Examples would be the Taroni-Bohm model with $\gamma=$ 1 and Erba models with $\gamma=5 / 2$.) The critical parameter $1 / \mathrm{L}_{\mathrm{c}}$ is found to have an offset-linear dependence on the magnetic shear parameter s/q [Eq. (5) and Fig. 3], showing the essential role of magnetic shear in electron transport. This magnetic shear dependence is consistent with the observation of improved energy confinement with increasing magnetic shear in the confinement zone with the high internal inductance mode of improved confinement in tokamaks. Jenko and Dorland [12] report that there is good agreement without the upshift shown here for a more complex ETG critical gradient evaluation for these Tore Supra discharges. A similar critical gradient analysis will need to be performed on other tokamaks under similar heating conditions to understand whether the upshift exists or is a result of modeling approximations.

\section{Acknowledgments}

This work was supported by the U.S. Dept. of Energy Contract No. DE-FG03-96ER-54346 and Association Euratom-CEA, CEA/DSM/DRFC. 


\section{REFERENCES}

[1] W. Horton, B.-G. Hong, and W. M. Tang, Phys. Fluids 31, 2971 (1988) and W. Horton, B. Hu, JQ. Dong, and P. Zhu, New J. Phys. 5, 14.1-14.34 (2003) (http://www.njp.org).

[2] P. N. Guzdar, C. S. Liu, J.-Q. Dong, and Y. C. Lee, Phys. Rev. Lett. 57, 2818 (1986).

[3] J.-Q. Dong, G. Jian, A. Wang, H. Sanuki, and K. Itoh, Nucl. Fusion 43, 183 (2003).

[4] T. S. Hahm and W. M. Tang, Phys. Fluids B 1, 1185 (1989).

[5] C. Bourdelle, X. Garbet, G. T. Hoang, and J. Ongena, Nucl. Fusion 42, 892 (2002).

[6] C. Fourment, G. T. Hoang, L.-G. Eriksson, X. Garbet, X. Litaudon, and G. Tresset, Plasma Phys. Control. Fusion 45, 33 (2003).

[7] A. M. Dimits, B. I. Cohen, W. M. Nevins, and D. E. Shumaker, et al., Phys. Plasmas 7, 969 (2000).

[8] Y. Idomura, S. Tokuda, and M. Wakatani, Phys. Plasmas 6(12), 4658 (1999).

[9] W. Dorland, F. Jenko, M. Kotschenreuther, and B. N. Rogers, Phys. Rev. Lett. 85(26), 5579 (2000).

[10] Jiquan, Li and Y. Kishimoto, Phys. Plasmas 10, 683 (2003); Phys. Plasmas 9, 1241 (2002).

[11] F. Jenko, W. Dorland, and G. W. Hammett, Phys. Plasmas 8(9), 4096 (2001).

[12] Jenko, F. and W. Dorland, Prediction of significant tokamak turbulence at electron gyroradius scales, Phys. Rev. Lett. 89, 22,5001 (2002). 
[13] Equipe Tore Supra, presented by B. Saoutic (Proceedings of Invited Papers, 21st European Conference on Controlled Fusion and Plasma Physics, Montpellier, 1994) Plasma Phys. Control. Fusion, Vol. 36 Suppl., B123 (1994).

[14] G. T. Hoang, B. Saoutic, L. Guiziou et al., Nucl. Fusion 38, 117 (1998).

[15] W. Horton, P. Zhu, G. T. Hoang, T. Aniel, M. Ottaviani, and X. Garbet, Phys. Plasmas 7, 1494 (2000).

[16] G. T. Hoang, C. Bourdelle, X. Garbet, G. Giruzzi, T. Aniel, M. Ottaviani, W. Horton, P. Zhu, and R. Budny, Phys. Rev. Lett. 87(12), 125001-1 (2001).

[17] G. T. Hoang, W. Horton, C. Bourdelle, B. Hu, X. Garbet, and M. Ottaviani, Analysis of the critical electron temperature gradient in Tore Supra, Phys. Plasmas 10(2), 604 (2003).

[18] B. B. Kadomtsev, Tokamak Plasmas: A Complex Physical System, (Institute for Physics Publishing, Bristol, 1992) pp. 124-150.

[19] A. Jacchia, F. De Luca, S. Cirant, et al., Gradient length driven electron heat transport study in modulated electron cyclotron heating FTU tokamak, Nucl. Fusion 42, 1116-1123 (2002).

[20] F. Ryter, G. Tardini, F. De Luca et al., Electron heat transport in ASDEX Upgrade: experiment and modeling, Nucl. Fusion 43, 1-9 (2003).

[21] P. Devynck, X. Garbet, C. Laviron, J. Payan, S. K. Saha, F. Gervais, P. Hennequin, A. Quemeneur, and A. Truc, Plasma Phys. Control. Fusion 35, 63 (1993).

[22] P. Devynck, F. Clairet, X. L. Zou, et al., Plasma Phys. Control. Fusion 39, 1355 (1997).

[23] X. L. Zou, L. Colas, M. Paume, J. M. Chareau, L. Laurent, P. Devynck, and D. Grésillon, Phys. Rev. Lett. 75, 1090 (1995). 
[24] L. Colas, X. L. Zou, M. Paume, J. M. Chareau, L. Guiziou, G. T. Hoang, Y. Michelet, and D. Grésilion, Nucl. Fusion 38, 903 (1998).

[25] K. L. Wong, K. Itoh, S.-I. Itoh, A. Fukuyama, and M. Yagi, Phys. Lett. A 276, 281-285 (2000).

[26] R. J. Hawryluk, S. Batha, W. Blanchard, et al., Phys. Plasmas 5, 1577 (1998).

[27] D. E. Kim, D-I. Choi, W. Horton, P. N. Yushmanov, and V. V. Parail, Transition from Neoclassical to Turbulent Electron Diffusion, Phys. Fluids B 2, 547-553 (1990).

[28] B. Labit and M. Ottaviani, Phys. Plasmas 10, 126 (2003).

[29] J.-Q. Dong, W. Horton, and Y. Kishimoto, Phys. Plasmas 8, 167 (2001).

[30] K. Itoh, S.-I. Itoh and A. Fukuyama: Transport and structural formation in plasmas (Institute for Physics Publishing, England, 1999).

[31] A. Bécoulet, D. Fraboulet, G. Giruzzi, D. Moreau, B. Saoutic, and J. Chinardet, Phys. Plasmas 1, 2908 (1994).

[32] F. Ryter, F. Leuterer, G. Pereverzev et al., Phys. Rev. Lett. 86, 2325 (2001).

[33] V. Basiuk, J. F. Artaud, F. Imbeaux, X. Litaudon, A. Bécoulet, L.-G. Eriksson, G. T. Hoang, G. Huysmans, D. Mazon, D. Moreau, and Y. Peysson, Simulations of steady-state scenarios for Tore Supra using the CRONOS code, Nucl. Fusion 43, 822-830 (2003).

[34] M. Uchida, A. Fukuyama, K. Itoh, S.-I. Itoh, and M. Yagi, Analysis of current diffusive ballooning mode including kinetic effects, Plasma Phys. Control. Fusion 44, 2495-2511 (2002). 
TABLE I: Linear stability theory critical temperature gradients in ETG and ITG

\begin{tabular}{ll}
\hline \hline Shearless Slab & $\left(\nabla \mathrm{T}_{\mathrm{e}}\right)_{\mathrm{c}}=2 \mathrm{~T}_{\mathrm{e}} / 3 \mathrm{~L}_{\mathrm{ne}}$ \\
& $\left(\nabla \mathrm{T}_{\mathrm{i}}\right)_{\mathrm{c}}=2 \mathrm{~T}_{\mathrm{i}} / 3 \mathrm{~L}_{\mathrm{ni}}$ \\
Sheared Slab & $\left(\nabla \mathrm{T}_{\mathrm{e}}\right)_{\mathrm{c}}=1.98 \mathrm{~T}_{\mathrm{e}}|\mathrm{s}|\left(1+\mathrm{Z}_{\mathrm{eff}} \mathrm{T}_{\mathrm{e}} / \mathrm{T}_{\mathrm{i}}\right) / \mathrm{qR}$ \\
& $\left(\nabla \mathrm{T}_{\mathrm{i}}\right)_{\mathrm{c}}=1.9 \mathrm{~T}_{\mathrm{i}}|\mathrm{s}|\left(1+\mathrm{T}_{\mathrm{i}} / \mathrm{T}_{\mathrm{e}}\right) / \mathrm{qR}$ \\
Flat Density Profile in a Torus & $\left(\nabla \mathrm{T}_{\mathrm{e}}\right)_{\mathrm{c}}=1.4 \mathrm{~T}_{\mathrm{e}}\left(1+\mathrm{Z}_{\mathrm{eff}} \mathrm{T}_{\mathrm{e}} / \mathrm{T}_{\mathrm{i}}\right) / \mathrm{R}$ \\
& $\left(\nabla \mathrm{T}_{\mathrm{i}}\right)_{\mathrm{c}}=1.4 \mathrm{~T}_{\mathrm{i}}\left(1+\mathrm{T}_{\mathrm{i}} / \mathrm{T}_{\mathrm{e}}\right) / \mathrm{R}$ \\
& $\left(\nabla \mathrm{T}_{\mathrm{e}}\right)_{\mathrm{c}}=\mathrm{T}_{\mathrm{e}} / \mathrm{R}\left(1+\mathrm{Z}_{\mathrm{eff}} \mathrm{T}_{\mathrm{e}} / \mathrm{T}_{\mathrm{i}}\right)(1.33+1.9|\mathrm{~s}| / \mathrm{q})$ \\
& $\left(\nabla \mathrm{T}_{\mathrm{i}}\right)_{\mathrm{c}}=\left(\mathrm{T}_{\mathrm{i}} / \mathrm{R}\right)\left(1+\mathrm{T}_{\mathrm{i}} / \mathrm{T}_{\mathrm{e}}\right)(1.1+1.4|\mathrm{~s}|+1.9|\mathrm{~s}| / \mathrm{q})$ \\
& $\left(\mathrm{R} / L_{T_{e}}\right)_{\mathrm{crit}}=\max \left\{\left(1+\tau_{\mathrm{i}}\right)(1.33+1.91 \hat{\mathrm{s}} / \mathrm{q})(1-1.5 \varepsilon)\right.$ \\
& $\left.(1+0.3 \varepsilon \mathrm{d} \kappa / \mathrm{d} \varepsilon), 0.8 \mathrm{R} / \mathrm{L}_{\mathrm{n}}\right\}$ \\
& \\
\hline \hline
\end{tabular}




\section{FIGURE CAPTIONS}

FIG. 1: Magnetic fluctuation $(\delta \mathrm{B} / \mathrm{B})^{2}$ at $\mathrm{k}_{\mathrm{r}}=12 \mathrm{~cm}^{-1}$ versus electron temperature gradient at midradius, measured by Cross Polarization Scattering for Tore Supra discharges $\left(\mathrm{I}_{\mathrm{p}}=1.3 \mathrm{MA}, \mathrm{B}=3.7 \mathrm{~T}\right.$, $\left.\mathrm{n}_{\mathrm{e}}(0)=6 \times 10^{19} \mathrm{~m}^{-3}\right)$ heated by Ion Cyclotron Resonance heating and Lower Hybrid waves $(1 \mathrm{MW}-$ 3.3MW).

FIG. 2: (a) Deviation of normalized FWEH heat flux $\mathrm{q}_{\mathrm{e}} / \mathrm{T}_{\mathrm{e}}^{\alpha}$ as a function of $\left(1 / L_{T_{e}}-1 / \mathrm{L}_{\mathrm{c}}\right)$ at $\mathrm{I}_{\mathrm{p}}=$ $0.6 \mathrm{MA}, \mathrm{B}=2.2 \mathrm{~T}$. (a) Relative deviation of the linear fit as a function of the exponent alpha for various normalized radii ranging from $\mathrm{r} / \mathrm{a}=0.2$ to $\mathrm{r} / \mathrm{a}=0.75$, for fixed density exponent $\beta=0$; (b) linear fit with $\alpha=3 / 2$; (c) relative deviation of the linear fit with $\alpha=3 / 2$ (solid), compared with the cases $\mathrm{q}_{\mathrm{e}} /\left(\mathrm{n}_{\mathrm{e}} \mathrm{T}_{\mathrm{e}}^{5 / 2}\right)$ (dashed) and $\mathrm{q}_{\mathrm{e}} /\left(\mathrm{n}_{\mathrm{e}} \mathrm{T}_{\mathrm{e}}^{3 / 2}\right)$ (dotted).

FIG. 3: a) Radial profile of $1 / \mathrm{L}_{\mathrm{c}}$ inferred from the power balance thermal flux extrapolated to zero flux compared with the ETG formula given by Eq. 6 (solid line); b) R/L $\mathrm{L}_{c}$ versus s/q (squares: power balance; circles: stability analysis; dashed line: fit).

FIG 4: Predictive simulation of an existing Tore Supra FWEH discharge $\left(\# 18368, \mathrm{I}_{\mathrm{p}}=0.6 \mathrm{MA}, \mathrm{B}=\right.$ $\left.2.2 \mathrm{~T}, \mathrm{n}_{\mathrm{e}}(0)=4 \times 10^{19} \mathrm{~m}^{-3}\right)$, with the fast wave power rising from $3 \mathrm{MW}$ to $6 \mathrm{MW}$. Time evolution of electron temperature $\left(\mathrm{T}_{\mathrm{e}}\right)$ at various radii, electron energy content $\left(\mathrm{W}_{\mathrm{e}}\right)$, loop voltage, and Faraday rotation angles (solid lines: simulations; dashed lines: experimental measurements). 


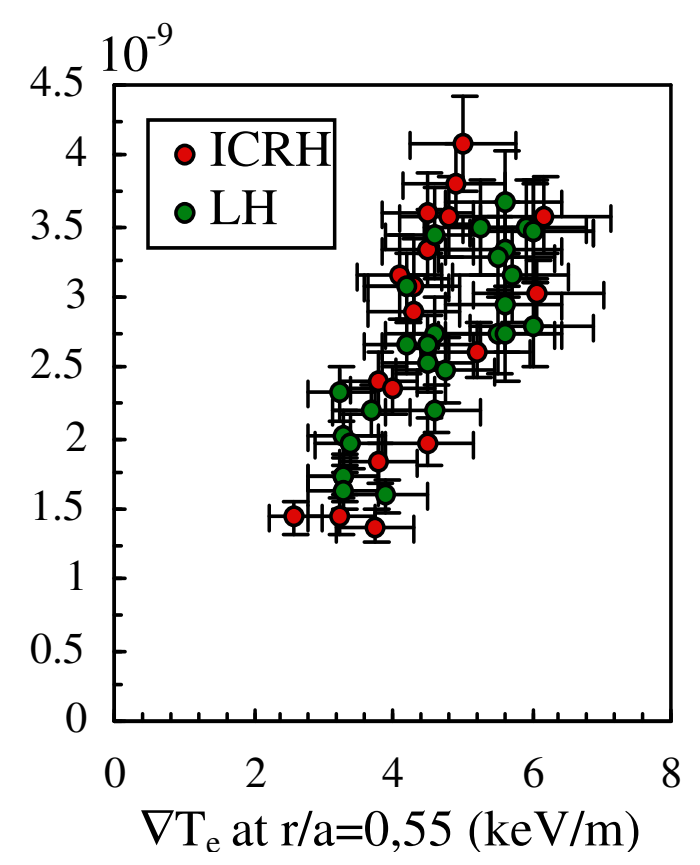

FIG.1
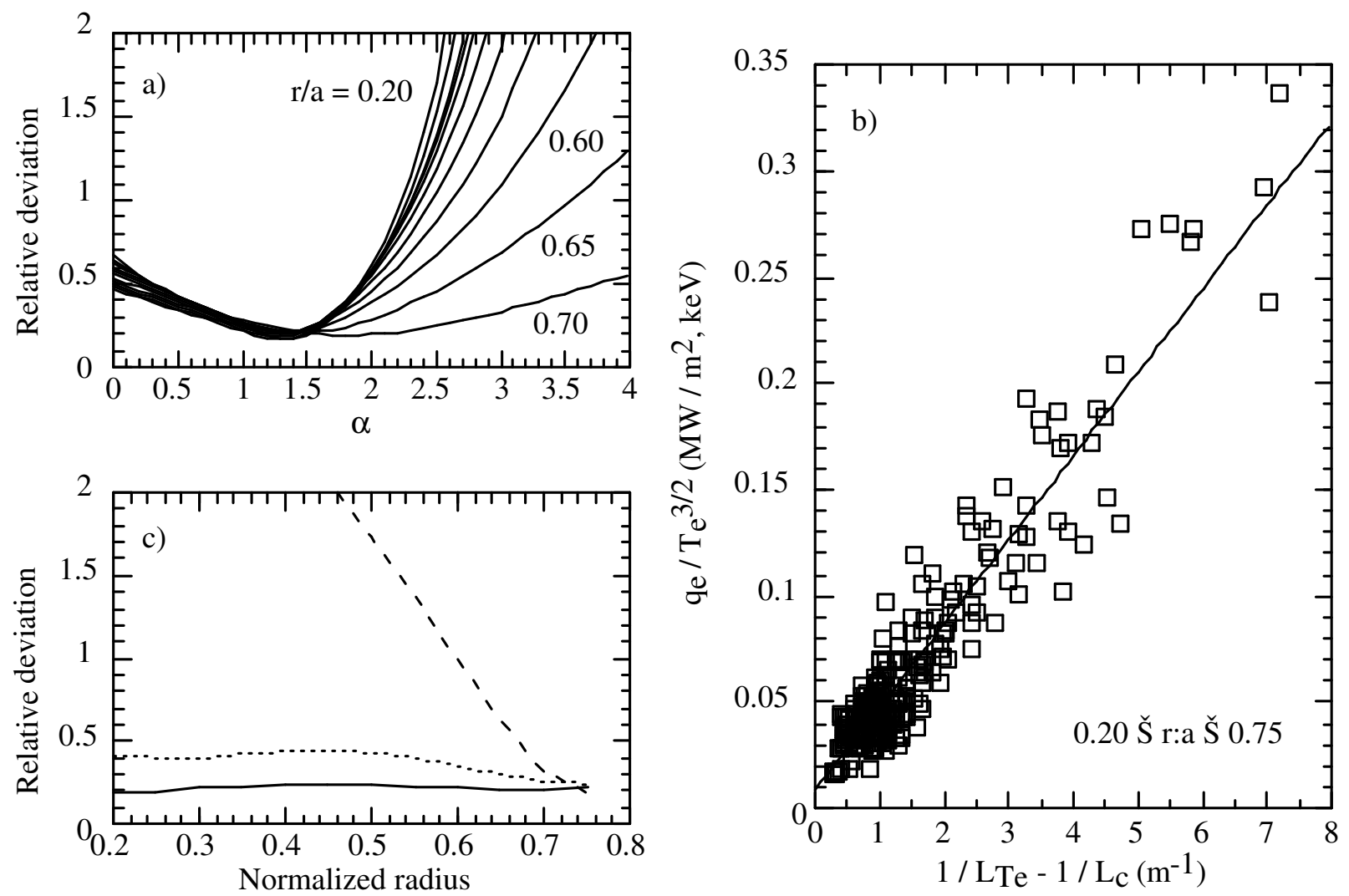
FIG. 2 
a)

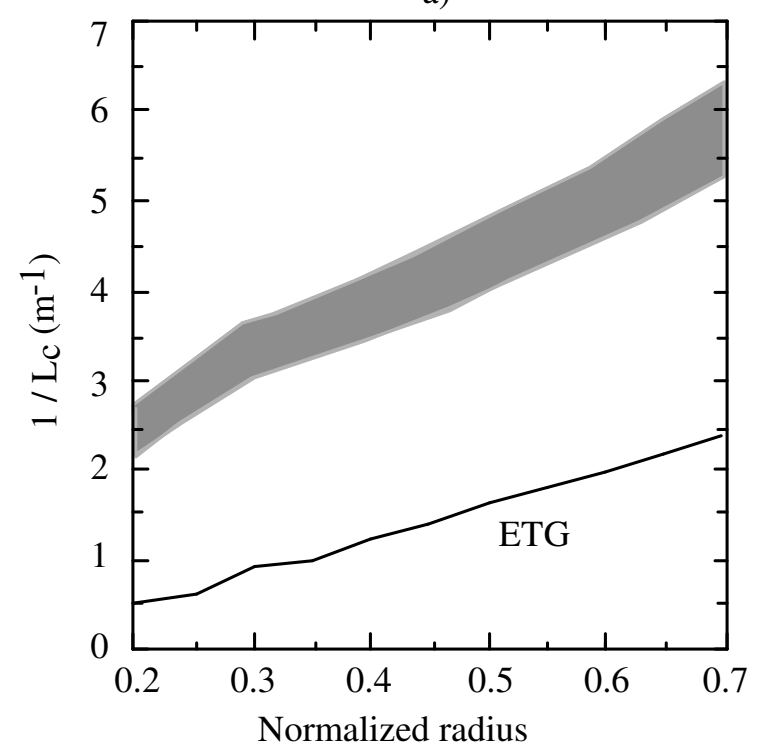

b)

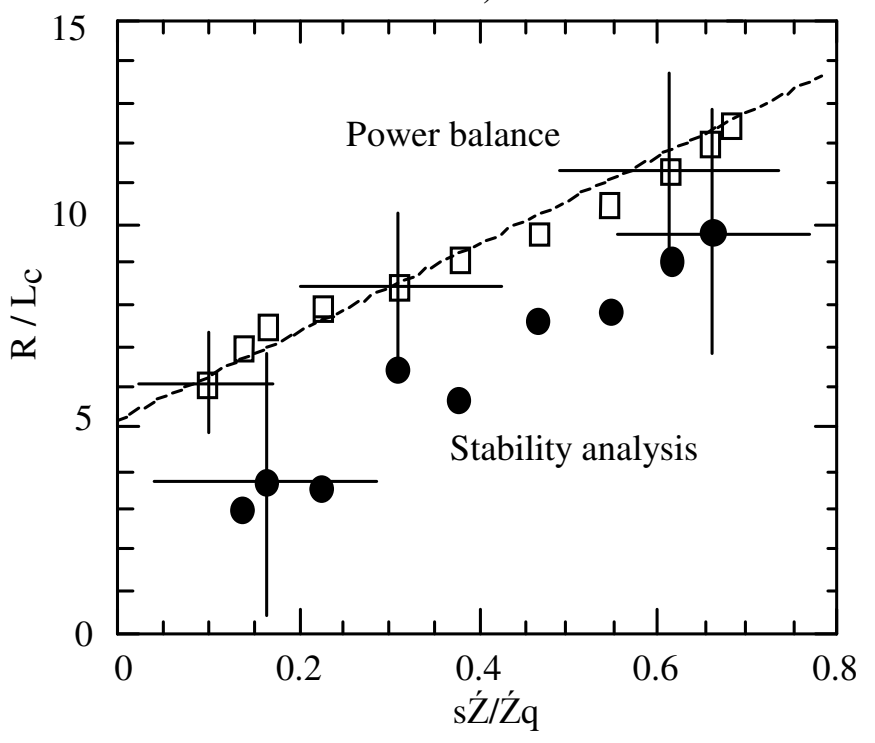

FIG. 3 

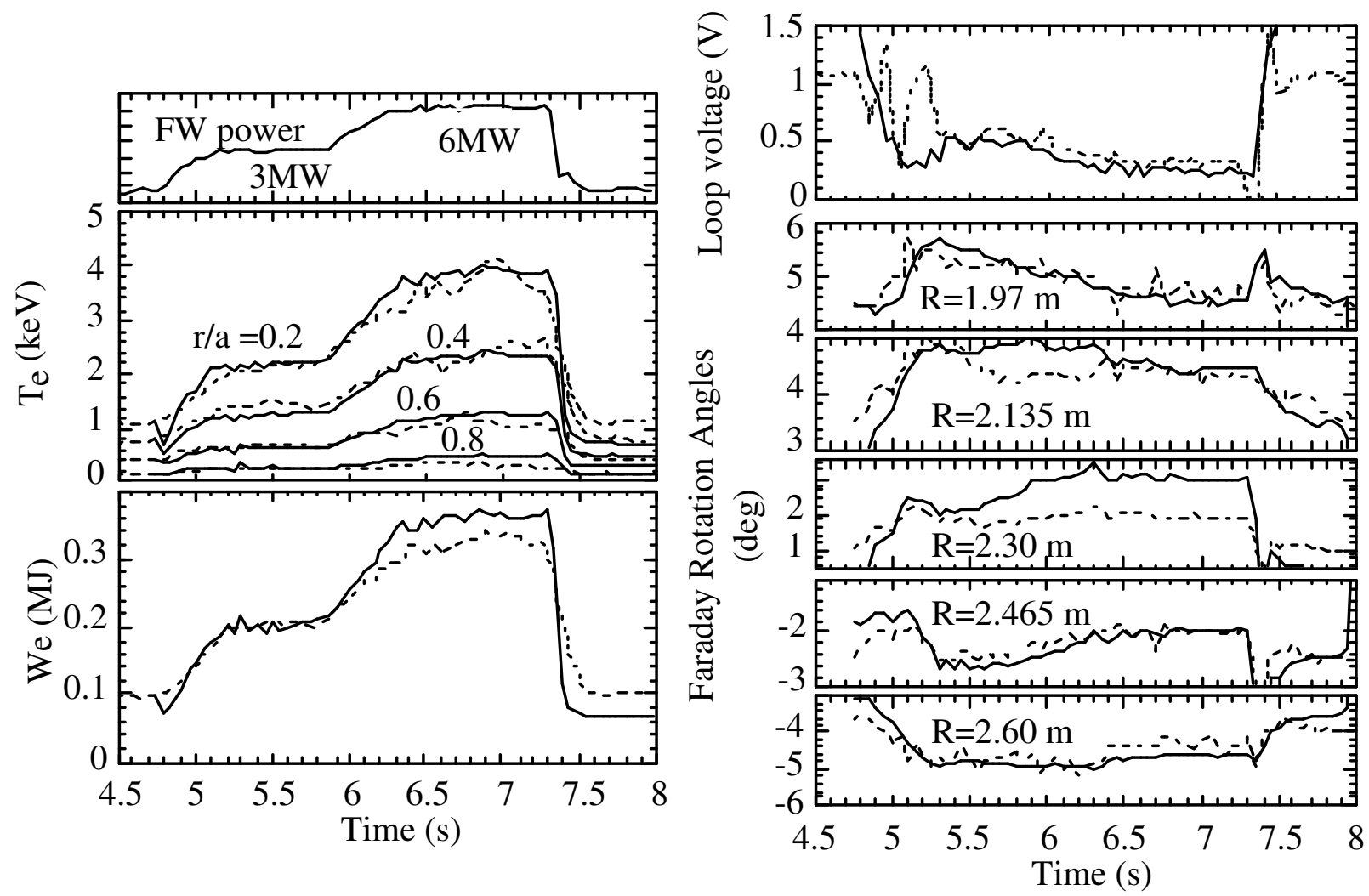

FIG. 4 